\title{
Structural modelling and analysis of Object Oriented Systems: a graph theoretic system approach
}

\author{
Nitin Upadhyay \\ Computer Science Group, \\ BITS-PILANI, Goa Campus, India \\ E-mail: upadhyay.nitin@gmail.com
}

\begin{abstract}
Object oriented methodology is becoming popular in the development of present day software. It is necessary to analyse architecture at the early stages of the development life cycle in order to avoid pitfalls in the quality of finished product. In the present work, a unitary system methodology for structural (architecture) modelling and analysis of Object-Oriented Systems (OOSs) is presented that describes the characteristics of performance, quality and reliability. The current work is an attempt to formulate computationally simple and analytical method to develop the structural design and analysis of OOS.
\end{abstract}

Keywords: graph theory; matrix approach; OOS; object-oriented system; system structure; VPF; variable permanent function.

Reference to this paper should be made as follows: Upadhyay, N. (2008) 'Structural modelling and analysis of Object Oriented Systems: a graph theoretic system approach', Int. J. Systems, Control and Communications, Vol. 1, No. 2, pp.240-255.

Biographical notes: Nitin Upadhyay is an acknowledged teacher and prolific writer. He is currently working as Faculty in the Computer Science and Information Systems Group, BITS, Pilani-Goa Campus. He has created a definite niche for himself in the field of Computer Science by contributing eight books. His research and creative zeal has enabled him to contribute research papers for journals and conferences. He is associated with various Journals of repute as Editor/Editorial Board Member/Reviewer. He is the recipient of Marquis Who's Who in the World, 2009 prestigious award. $\mathrm{He}$ is a member of various prestigious professional societies such as IEEE, IEEE Computer Society, IEEE Reliability Society, IEEE Communication Society, ACM and Computer Society of India. His major research areas are COTS-based system development, COTS selection, evaluation and certification, software engineering and software architecture, system engineering, concurrent engineering, quality modelling and analysis, object oriented system, graph theory, mobile learning and mode of education.

\section{Introduction}

In recent years we have noticed the growing impact of the object-orientation paradigm.It is widely accepted that object oriented development requires a different way of thinking than traditional structured development (Börstler, 2002). An Object-Oriented System (OOS) is an organised collection of cooperative objects representing real world 
entities. It has been identified by industry and academia that investing in architecture design in the early stage of lifecycle is of paramount importance to a project's success (Bosch, 2000; Clements et al., 2002; Kruchten, 1995; Shaw and Garlan, 1996) Researchers have identified that the performance of any system is a function of its basic architecture (i.e., layout and design) (Wilkinson and Byers, 1993). A structure is the set of sub-systems/components and interconnections within and across them. The understanding of systems architecture and its connectivity and interactions between different systems, and down to component level, is useful for estimating the contribution of different attributes of the performance of the system. In object idiom, assemblies of components are called composite objects (Rumbaugh, 1994). The structure of composite objects consists of its components and interconnections within and across them. Systems engineering decompose a system into a collection of sub-systems that participate in collaboration to meet the desired product function (Kriokorian, 2003). A decomposition of the system involves an identification of the concepts, attributes, and associations that are considered significant (Coad and Yourdon, 1991). An OOS is decomposed into sub-systems and sub-sub-systems. A system hierarchy represents the overall system composition. The importance of decomposition of system in analysing large and complex systems has been observed (Courtois, 1985). The performance of a complete OOS (e.g., quality, availability, reliability) depends upon the performance of its macro-level systems and interconnections in an integrated manner. Currently, no effective mathematical model is present for studying these aspects in relation to each other or independently.

This proposed methodology would permit the researchers, academicians, designers, analysts and developers to design, analyse, develop and improve their products. Various strategic and marketing decisions based on the competitive market can also be taken by comparing available architectures/products by the methodology as specified. This is achieved with the help of graph theory, matrix-algebra, and permanent function. This tool has so far been used by various authors to study a sub-system for a particular attribute of the performance of a system in thermal power plants (Mohan et al., 2003), nuclear plants (Sacks et al., 1983), selection of rolling elements of bearings (Seghal et al., 2000), maintainability index (Gandhi et al., 1991), but so far it has not been used to model and analyse an OOS.

\section{Identification of system}

A top-level system is viewed as a combination of various systems and sub-systems. The structure of OOS is dependent on the elements contained in the boundary and their interconnections. In order to perform the complete design and analysis of an OOS, we also have to consider contributing factors other than the main physical sub-systems and their interconnections. A sub-system is a system in itself. A decomposition criterion is used to decompose the system into subjects. The most frequently used decomposition criteria for system are defined below (Tagoug, 2002):

- data criterion: classes sharing same data.

- business function criterion: classes contributing to achieve same business function.

- time criterion: classes instantiated in the same slice time. 
- organisational structure criterion: classes belonging to the same organisational unit.

- behaviour criterion: classes affected by change of state in some class.

An example of the decomposition process to develop OOS decomposition model is shown in Figure 1.

Figure 1 Decomposition of Object Oriented System

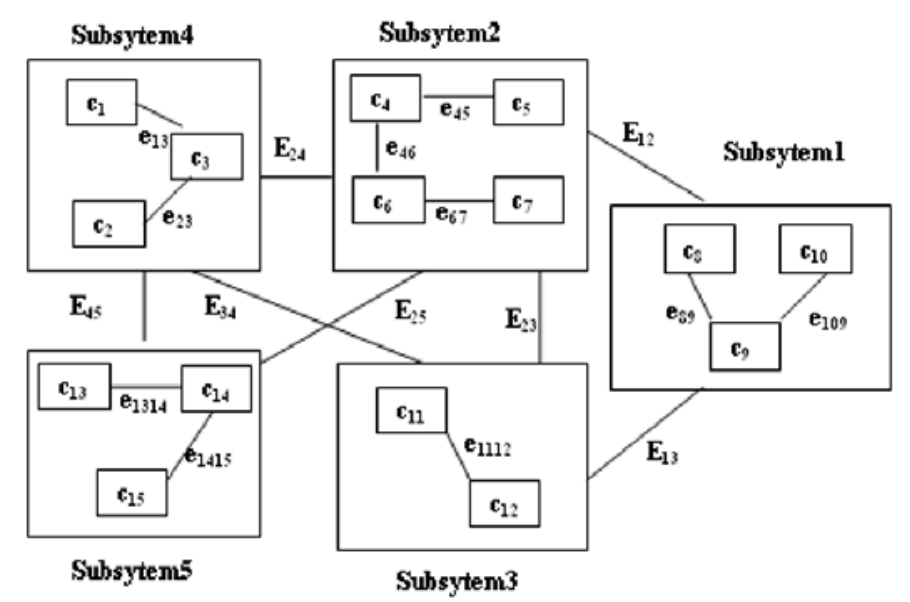

The OOS decomposition model consists of the following:

- Sub-systems. A (sub) system connected to another (sub) system in a larger system. For example, sub-sytem 1 is connected to sub-system 2 and sub-sytem 3 .

- Interactions. Physical or conceptual (within or across). A physical interaction represents a message connection or instance connection or inheritance (generalisation specialisation structure) or a whole part structure. For example there is message connection between class $C_{4}$ and $C_{5}$, represented as $\mathrm{e}_{45}$. There is a conceptual connection between two classes if they share some property. For example, there is data conceptual connection between class $\mathrm{C}_{4}$ and $\mathrm{C}_{6}$, represented as $\mathrm{e}_{46}$. The connection between sub-systems can be represented in two ways: a single connection, for example, $\mathrm{E}_{12}$ (connection between sub-system 1 and sub-system2); multiple connections between inter-system class connection; for example, $\mathrm{E}_{12}$ can be decomposed into four message connections as $c_{49}, c_{59}, c_{68}$, and $c_{69}$. Interactions $T_{i j}$ can be written as a function of basic parameters involved in connection. The general form is $T_{i j}=f$ ([physical or conceptual connections], dependency) $\left\{T_{i j}\right.$ is interaction between sub-systems $i$ and $\left.j\right\}$

$$
T_{12}=f\left(\left[c_{49}, c_{59}, c_{68}, c_{69}\right],\left[c_{49} \rightarrow c_{59}, c_{68} \rightarrow c_{69}\right]\right) \text {. }
$$

To define an OOS engineering process, an outline of the necessary tools and procedure to support it is required. Initially, system requirement is identified, which is broken down for further analysis, generating its own set of requirements. The whole process is repeated, containing a more detailed view of the system and sub-systems, until the collapsed component level is reached. The prime objective of system approach is to 
facilitate through evaluation and proper accommodation of new concepts and technology in an OOS model.

Industry is free to identify a set of sub-systems as per its requirements, aims and objectives. To understand the function and performance of OOS, the interaction and interdependency from the point of view of business, researchers, maintenance etc, have to be analysed.

\section{Graph theoretic modelling of Object Oriented System}

A system graph $G=[S, E]$ is used to model system architecture by applying graph theory. Here $S\left(S_{i}=1,2, \ldots, n\right)$, the vertex set, denotes the set of sub-systems and $E\left(E_{i}=1\right.$, $2, \ldots, n)$, the edge set, denotes the interconnections between sub-systems of set $S$. The OOS represented by system structure graph (SSG) is called the Object Oriented System Structure Graph (OOSSG). The connections and interconnections vary depending upon the type of edge used. The undirected edges show the connectivity between (sub) systems or components and the directed edges represent the flow of information or interaction or dependency.

The OOSSG of Figure 1 is shown in Figures 2 and 3. The five nodes represent the respective systems of $O O S$ and edges corresponding to the connections/interactions between the sub-systems. A graph theory based architecture model is used to represent direct, undirected or hybrid interactions between sub-systems. A real life OOS is represented graphically by directed graph Figure 2 and undirected graph Figure 3.

Figure 2 OOSSG directed graph

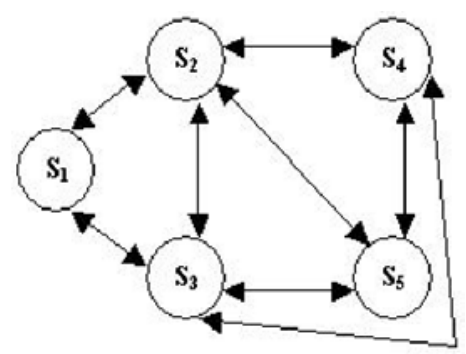

Figure 3 OOSSG undirected graph

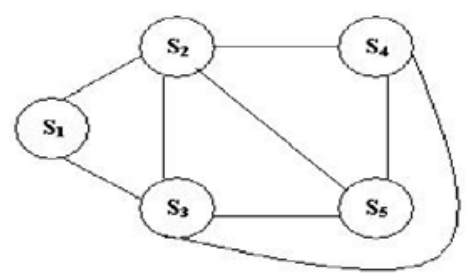

The connectivity may be directed or undirected depending upon the structural, functional or performance considerations. Depending upon various design aspects, strategic, marketing and user centric decisions the proposed methodology update, modify and delete systems or sub-systems. For visual analysis and understanding the proposed SSG 
representation is suitable, but it is not appropriate for computer processing. If the number of systems is more, then the overall system becomes more complex for understanding and visual analysis. Moreover, upon changing, labels of vertices/systems result in a new SSG. In view of this, we present a computer efficient representation. Many matrix representations are available in the literature (Deo, 2004; Upadhyay, 2004), for example, adjacency and incidence matrices. The adjacency matrix is a square matrix and used for this purpose. Using this, the OOS is represented in matrix form.

\subsection{Variable Permanent System Structure Matrix (VPSSM - OOS)}

In order to describe proper characterisation of the OOS as derived from combinatorial considerations, a permanent matrix P, is proposed (Jurkat and Ryser, 1996). The matrix function/permanent $\operatorname{Per}(P)$ of VPSSM - OOS is capable of describing a whole OOS i.e., system graph in a single multinomial equation. Let the off-diagonal elements matrix $F$ consist of $E_{i j}$ to represent interaction/connectivity (system ' $i$ ' is connected to system ' $j$ ') and also $E_{i j}=E_{j i}$. Let us also define diagonal matrix $D$ with its variable diagonal elements $S_{i}(i=1,2, \ldots, 5)$ representing the characteristic structure features of five distinct systems. Let the complete permanent matrix of five-sub-system OOS with all possible interactions present be defined as

$$
\begin{aligned}
& \begin{array}{llllll}
1 & 2 & 3 & 4 & 5 & \text { Sub-system }
\end{array} \\
& \text { a } S_{1} \quad E_{12} \quad E_{13} \quad E_{14} \quad E_{15} \text { ㅇ } 1
\end{aligned}
$$

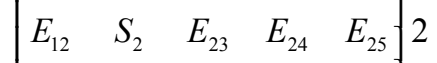

$$
\begin{aligned}
& P=\left\langle E_{13} \quad E_{23} \quad S_{3} \quad E_{34} \quad E_{35} » 3 .\right. \\
& \begin{array}{lllll}
\ll E_{14} & E_{24} & E_{34} & S_{4} & E_{45} »
\end{array} \\
& \begin{array}{llllll} 
& \ll E_{15} & E_{25} & E_{35} & E_{45} & S_{5} \gg / 2
\end{array}
\end{aligned}
$$

A Variable Permanent System Structure Matrix (VPSSM - OOS) ' $V_{p}$ ' of SSG with $E_{i j}=E_{j i}$ in Figure 3 is written as:

$$
\begin{aligned}
& V_{p}=\{D+F\} \\
& \begin{array}{llllll}
1 & 2 & 3 & 4 & 5 & \text { Sub-system }
\end{array}
\end{aligned}
$$

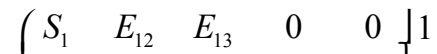

$$
\begin{aligned}
& \begin{array}{llllll}
\ll E_{12} & S_{2} & E_{23} & E_{24} & E_{25}{ }^{»} 2
\end{array} \\
& V_{P}=\ll E_{13} \quad E_{23} \quad S_{3} \quad E_{34} \quad E_{35} » 3 . \\
& \begin{array}{lllll}
\ll 0 & E_{24} & E_{34} & S_{4} & E_{45} » 4
\end{array}
\end{aligned}
$$

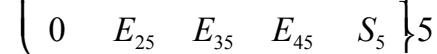

It is a complete representation of an OOS, as it does not contain any negative sign. This means that it preserves all the structural information about dyads, loops of systems, or system attributes such as reliability, availability etc even in numerical form. The single characteristics multinomial equation based on VPSSM-OOS is known as a Variable Permanent Function (VPF) of OOS. The VPF - OOS for matrix is written as: 


$$
\begin{aligned}
\operatorname{Per}\left(V_{p}\right)= & S_{1} S_{2} S_{3} S_{4} S_{5}+\left[E_{12}^{2} S_{3} S_{4} S_{5}+E_{13}^{2} S_{2} S_{4} S_{5}+E_{23}^{2} S_{1} S_{4} S_{5}\right. \\
& +E_{24}^{2} S_{1} S_{3} S_{5}+E_{25}^{2} S_{1} S_{3} S_{4}+E_{34}^{2} S_{1} S_{2} S_{5}+E_{35}^{2} S_{1} S_{2} S_{4} \\
& \left.+E_{45}^{2} S_{1} S_{2} S_{3}\right]+\left[2 E_{12} E_{23} E_{31} S_{4} S_{5}+2 E_{23} E_{34} E_{42} S_{1} S_{5}\right. \\
& \left.+2 E_{23} E_{34} E_{45} S_{1} S_{4}+2 E_{24} E_{45} E_{52} S_{1} S_{3}+2 E_{34} E_{45} E_{53} S_{1} S_{2}\right] \\
& +\left\{\left[2 E_{23} E_{34} E_{45} E_{52} S_{1}+2 E_{23} E_{35} E_{54} E_{42} S_{1}+2 E_{24} E_{43} E_{35} E_{52} S_{1}\right.\right. \\
& \left.+2 E_{12} E_{24} E_{43} E_{31} S_{5}+2 E_{12} E_{25} E_{53} E_{31} S_{4}\right]+\left[E_{13}^{2} E_{45}^{2} S_{2}+E_{24}^{2} E_{35}^{2} S_{1}\right. \\
& +E_{25}^{2} E_{13}^{2} S_{4}+E_{24}^{2} E_{13}^{2} S_{5}+E_{23}^{2} E_{45}^{2} S_{1}+E_{25}^{2} E_{34}^{2} S_{1}+E_{12}^{2} E_{45}^{2} S_{3} \\
& \left.\left.+E_{12}^{2} E_{34}^{2} S_{5}+E_{12}^{2} E_{35}^{2} S_{4}\right]\right\}+\left\{\left[2 E_{12}^{2} E_{34} E_{45} E_{53}+2 E_{45}^{2} E_{12} E_{23} E_{31}\right.\right. \\
& \left.\left.+2 E_{13}^{2} E_{24} E_{45} E_{52}\right]+\left[2 E_{12} E_{24} E_{45} E_{53} E_{31}+2 E_{12} E_{25} E_{54} E_{43} E_{31}\right]\right\}
\end{aligned}
$$

Irrespective of labelling of sub-systems the above equation (multinomial) uniquely represents the OOS of Figure 1. Every term of these equations represents a sub-set of the OOS. It is possible to write these equations simply by visual inspection of the OOS of Figures 2 and 3, as every term corresponds to a physical sub-system of the complete system. To achieve this objective, the permanent function of equation (3) is written in a standard form as $(N+1)$ groups. All these distinct combinations of sub-systems and interactions of the macro-system are shown graphically in Figure 4.

Figure 4 Graphical/physical representation of permanent function expression of OOS

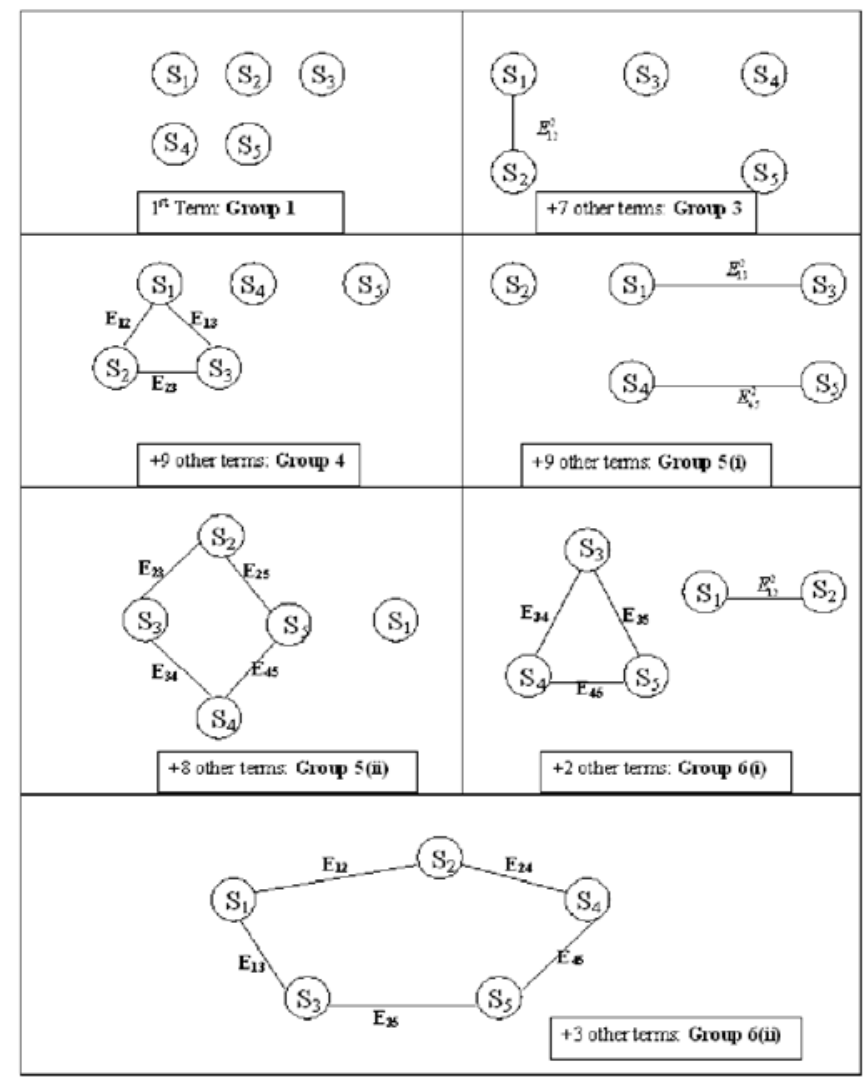


The multinomial, i.e., the permanent function when written in $(N+1)$ groups, presents an exhaustive way of analysis of OOS at different levels. It helps in identifying different critical components and links to improve reliability, fault tolerance, performance, quality, security, autonomy and availability of system.

On critical analysis of permanent function (3) it is inferred that this multinomial contains only distinct sub-systems $-S_{i}$, dyads $-E_{i j}^{2}$ and loops $-E_{i j}, E_{j k}, \ldots, E_{n i}$. A complete permanent function has been written in a systematic manner for unambiguous and unique interpretation. In short, it can be represented as:

$$
\begin{aligned}
\operatorname{Per}\left(V_{P}\right) & =g\left(S_{i,}, E_{i j}^{2}, E_{i j}, E_{j k}, E_{k i} \text { etc. }\right) \quad\left\{\text { if } E_{i j}=E_{j i}\right\} \\
& =g(\text { Vertices, dyads, loops }) \\
& =g \text { (structural components }) \\
\operatorname{Per}\left(V_{P}\right) & =g^{\prime}\left(S_{i,}, E_{i j} E_{j i}, E_{i j} E_{j k} E_{k l} E_{l i}, E_{i j} E_{j k} E_{k l} E_{l m} E_{m i}\right) \quad\left\{\text { if } E_{i j} \neq E_{j i}\right\} \\
& \left.=g^{\prime} \text { (Vertices, 2-vertex loops, loops }\right) \\
& =g^{\prime} \text { (structural components). }
\end{aligned}
$$

The terms of the permanent function $\operatorname{Per}\left(V_{P}\right)$ are arranged in $(N+1)$ groups in decreasing order of the number of vertices/sub-systems $S_{i}$ present in each term. The first group contains terms with $(N-1) S_{i}$ 's. the second group will contain terms with $(N-2) S_{i}$ 's and remaining as dyad $E_{i j}^{2}$ or $E_{i j} E_{j i}$ and so on. The last group does not contain any $S_{i}$ in its terms. It contains only terms such as $E_{i j}^{2}, E_{i j} E_{j k} E_{k i}$, etc.

- Group 1. The first term (grouping) represents a set of $N$ unconnected OOS sub-systems, i.e., $S_{1}, S_{2}, \ldots, S_{N}$.

- Group 2. This group is absent as a particular sub-system has no interaction with itself (absence of self-loops) i.e., any of the sub-system is not connecting itself.

- Group 3. Each term of the third grouping represents a set of two-element OOS loops (i.e., $\left.S_{i j} S_{j i}\right)$ and is the resultant OOS dependence of characteristics $i$ and $j$ and the OOS measure of the remaining $(N-2)$ unconnected elements/sub-systems. A group has eight terms, each term is a set of one dyad, $E_{i j}^{2}$ or a two-sub-system loop i.e., $E_{i j} E_{j i}$ and three independent sub-systems (dyad is a system of two sub-systems $i$ and $j$, considered as one entity).

- Group 4. Each term of the fourth grouping represents a set of three-element OOS sub-system interaction loops $\left(E_{i j} E_{j k} E_{k i}\right.$ or its pair $\left.E_{k j} E_{j i}\right)$ and the composite system measure of the remaining $(N-3)$ unconnected elements. A group has $(2 * 5) 10$ terms in all. Each term has a set of one 3-sub-system loop $\left(e_{i j} e_{j k} e_{k i}\right)$ and independent sub-systems. The three-sub-system loop is a system, to be considered as one entity.

- Group 5. The fifth grouping contains two sub-groups. The terms of the first sub-grouping consist of two-element OOS sub-system interaction loops (i.e., $E_{i j} E_{j i}$ and $E_{k l} E_{l k}$ ) and OOS constituent $E_{m}$. The terms in the second grouping are the product of four-element OOS sub-system interaction loops (i.e., $E_{i j} E_{j k} E_{k l} E_{l i}$ ) or its pair (i.e., $E_{i l} E_{l k} E_{k j} E_{j i}$ ) and OOS constituent $S_{m}$. A group has two sub-groups: 
- Group 5(i) has ten terms; each term is a sub-set of two independent dyads $\left(E_{i j}^{2}, E_{k l}^{2}\right)$ or two-sub-system loops and one independent sub-system.

- Group 5(ii) has nine terms; each term is a set of four-sub-system loop $\left(E_{i j} E_{j k} E_{k l} E_{l i}\right)$ and one independent sub-system.

- Group 6. The terms of the sixth grouping are also arranged in two sub-groupings. The terms of the first sub-grouping are a product of a two-element OOS sub-system interaction loop (i.e., a three-element OOS sub-system interaction loop (i.e., $E_{k l} E_{l m}$ $E_{m k}$ )or its pair (i.e., $E_{k m} E_{m l} E_{l k}$ ). The second sub-grouping consists of a five-component OOS sub-system interaction loop (i.e., $E_{i j} E_{j k} E_{k l} E_{l m} E_{m i}$ )or its pair $\left(E_{i m} E_{m l} E_{l k} E_{k \mathrm{j}} E_{j i}\right)$. A group has again two sub-groups:

- Group 6(i) has one three-sub-system loop and a dyad or two-sub-system loop while

- Group 6(ii) has three five-sub-system loops.

By providing/associating proper physical meaning to the VPF-OOS structural components, appropriate interpretation is obtained: $E_{i j}^{2}$ is interpreted as a two-system structural dyad, for example, $E_{35}^{2}$ represents the dyad of interaction between sub-system $S_{3}$ and $S_{5}$.

$E_{i j} E_{j k} E_{k i}$ is a three system structural loop; for example, $E_{12} E_{23} E_{31}$ represents the three system structural loop between $S_{1}, S_{2}$ and $\mathrm{S}_{3}$ systems.

$E_{i j} E_{j k} E_{k l} E_{l i}$ is a four system structural loop; for example, $E_{23} E_{34} E_{45} E_{52}$ represents four-system structural loop, between $S_{2}, S_{3} S_{4}$, and $S_{5}$ systems.

In all, a general five-sub-system permanent function will have 5! i.e., 120 terms (sub-sets) arranged in $(N+1)$ groups. Figure 4 gives graphical/physical interpretation of terms of different groups for visual understanding, analysis, and improvement of OOS architecture. It is therefore possible for the system analyst and designer to carry out SWOT (strength-weakness-opportunities-threats) analysis of their complete OOS and take strategic decisions to their advantage as per policy. The permanent function $\operatorname{Per}\left(V_{p}\right)$ defines OOS System index when numerical values for different parameters (quality, reliability etc.) are substituted in the equation.

\section{Evaluation of $V_{P}$}

The diagonal elements of the matrix in equation (2) correspond to the five sub-systems that constitute a OOS. The values of these diagonal elements $S_{1}, S_{2}, \ldots, S_{5}$ are calculated as:

$$
S_{1}=\operatorname{Per}\left(V_{P} S_{1}\right) \quad S_{2}=\operatorname{Per}\left(V_{P} S_{2}\right) \quad S_{3}=\operatorname{Per}\left(V_{P} S_{3}\right) \quad S_{4}=\operatorname{Per}\left(V_{P} S_{4}\right) \quad S_{5}=\operatorname{Per}\left(V_{P} S_{5}\right)
$$

where $V_{P} S_{1}, V_{P} S_{2}, V_{P} S_{3}, V_{P} S_{4}, V_{P} S_{5}$ are the variable permanent matrices for five sub-systems of the OOS. The procedure for calculating $S_{1}, S_{2}, \ldots, S_{5}$ is the same as for calculating $\operatorname{Per}\left(V_{P}\right)$ of equation (3). For this purpose, the sub-systems of an OOS are considered, and the procedure given below is followed: 
- The decomposition model of these sub-systems is drawn separately by considering their various sub-subsystems.

- Identify the degree of interactions, interconnections, dependencies, connectivity, etc. between different sub-sub-systems.

Digraph representations (like Figure 2) of five sub-systems are drawn first separately to obtain their matrix equations (like equation (3)) i.e., $V_{P} S_{i}$ and then their permanent functions $\operatorname{Per}\left(V_{P} S_{i}\right), S_{i,} i=1, \ldots, 5$. The off-diagonal terms $e_{i j}(i, j=1,2, \ldots, 5)$ of matrix equation (2) give the connections between the systems $S_{i}$ and $S_{j}$. Depending upon the type of structural analysis, $S_{i j}$ can be represented as multinomial, graph, and matrix or by some analytical model. To get the exact degree of interactions, interconnections, dependencies, connectivity, etc. between sub-systems or sub-subsystems we may have to consider the views of technical team experts. A team of experts has to consider all the issues involved from the point of view of engineering, science, technology, and business strategy. The final decision on the values of $S_{i}$ and $S_{i j}$ may be taken on the recommendations of the team. Thus, following the top-down approach and the step-by-step procedure given below will give the complete structural analysis of the OOS.

\section{Compact representation of permanent function}

The VPF (VPSSM - OOS) being the characteristic of OOS of any industrial product is a powerful tool for its evaluation and analysis. The VPSSM - OOS system expression, which corresponds to the five-factor digraph and matrix, equation (1), is written in a compact sigma (1 ) form.

$$
\begin{aligned}
& \operatorname{Per}\left(V_{P}\right)=\prod_{1}^{5} S_{i}+\mathbf{i}_{i} \quad \mathbf{i}_{j} \quad \mathbf{i}_{k} \quad \mathbf{i}_{l} \mathbf{i}_{m}\left(E_{i j} E_{j i}\right) S_{k} S_{l} S_{m} \\
& +\mathbf{i}_{i} \mathbf{I}_{j} \mathbf{I}_{k} \mathbf{I}_{l} \mathbf{i}_{m}\left(E_{i j} E_{j k} E_{k i}+E_{i k} E_{k j} E_{j i}\right) S_{l} S_{m}
\end{aligned}
$$

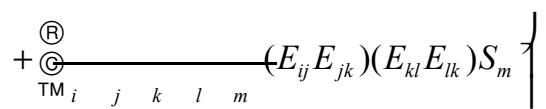

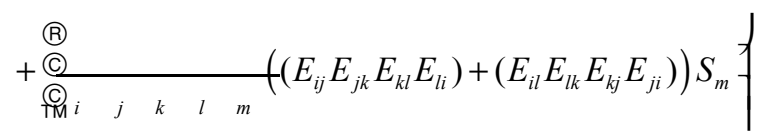

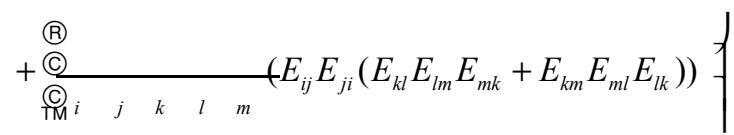

$$
\begin{aligned}
& +\mathbf{I}_{i} \mathbf{I}_{j} \mathbf{I}_{k} \mathbf{I}_{l} \mathbf{I}_{m}\left(E_{i j} E_{j k} E_{k l} E_{l m} E_{m i}+E_{i m} E_{m l} E_{l k} E_{k j} E_{j i}\right) .
\end{aligned}
$$

The above equation is a generalised mathematical expression in symbolic form corresponding to five-factor digraph representation. It ensures an estimate of the OOS of any industrially integrated product. The above equation contains 5 ! terms. Each term is useful for a OOS designer as each term serves as a test for the effectiveness of the relevant group in $\operatorname{Per}\left(V_{P}\right)$. 


\section{Generalising methodology}

If a system consists of $N$ sub-systems and is represented as a digraph, then the most general way of matrix representation is shown below. This matrix is also known as the variable permanent matrix (VPSSM- OOS) corresponding to the $N$ sub-systems.

$$
\begin{aligned}
& \begin{array}{llllll}
1 & 2 & 3 & 4 & 5 & \text { Sub-system }
\end{array}
\end{aligned}
$$

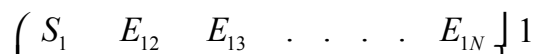

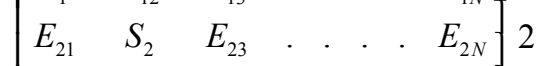

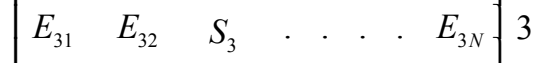

$$
\begin{aligned}
& \ll \text {. } \\
& \ll, \cdot \text {. } \\
& \ll \cdot \text {. }
\end{aligned}
$$

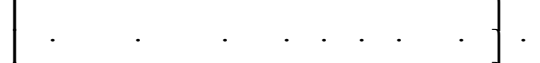

$$
\begin{aligned}
& \begin{array}{lllllll}
\ll E_{N 1} & E_{N 2} & E_{N 3} & . & . & S_{N} & \Downarrow / 4
\end{array}
\end{aligned}
$$

Permanent for the above matrix, i.e., Per $\left(V_{P}\right)$ is called VPF (VPSSM - OOS). The VPSSM - OOS for the above matrix is written in sigma form as

$$
\begin{aligned}
& \operatorname{Per}\left(V_{p}\right)=\prod_{x=1}^{N} S_{x}+\mathbf{i}_{i} \mathbf{I}_{j} \mathbf{I}_{k} \mathbf{I}_{l} \ldots \mathbf{i}_{N}\left(E_{i j} E_{j i}\right) S_{k}, S_{l}, \ldots, S_{N}
\end{aligned}
$$

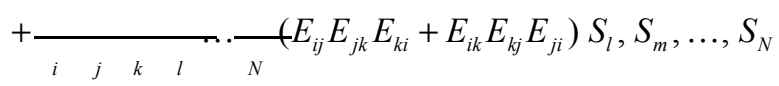

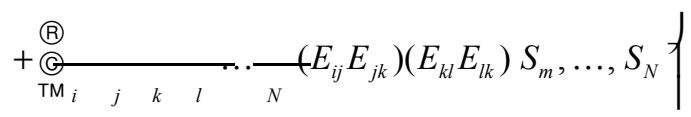

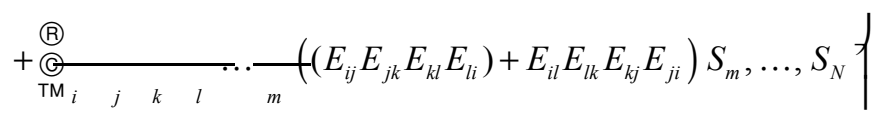

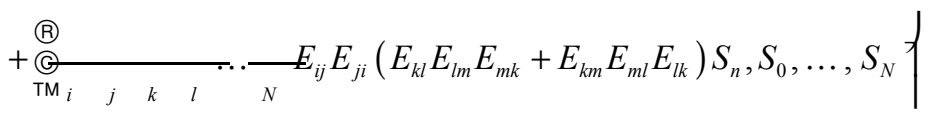

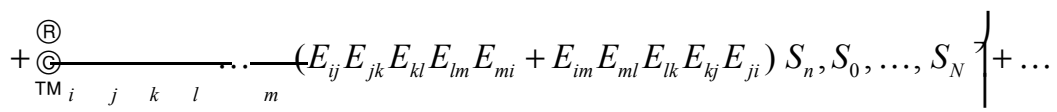

The number and composition of groups and sub-groups will be the same as discussed earlier. So it is possible to write the permanent function of any OOS in $(N+1)$ groups. It may be noted that a permanent function will contain $N$ ! terms only, provided $e_{i j}$ are not 0 . In certain cases, designers and/or developers team may decide that some of $e_{i j}$ are 0 because of the insignificant influence of one sub-system over the other sub-system. Substitutions of corresponding $e_{i j}$ equal to 0 in general permanent function (equation (7)) or in general VPM (equation (6)) give the exact number of terms with the modified permanent function. 


\section{Identification and comparison of Object Oriented System architecture}

OOS architecture composition (sub-systems) affects the properties and performance of the finished product. The five-sub-systems of an OOS, Figure 1, are modelled as a multinomial, a permanent function. Different systems developed using different sub-systems and technologies will have a different number of terms in different groups and sub-groups of their permanent functions because of change in structure and interactions. The similarity or dissimilarity in the structure between two OOSs is obtained by comparing their permanents. Using the proposed methodology, the identification of OOS architecture and its comparison with other OOS architecture is based on the analysis carried out with the help of VPF - OOS. Two OOS architectures are similar to sub-systems and their interactions only if their digraphs are isomorphic. Two OOS architecture digraphs are isomorphic if they have identical VPF - OOS. This means that the set of the number of terms in each grouping/sub-grouping of two OOSs is the same. Based on this, an OOS identification set for any product is written as:

$$
\left[\left(I_{1} / I_{2} / I_{3} / I_{4} / I_{51}+I_{52} / I_{61}+I_{62} / \ldots\right)\right]
$$

where $I_{i}$ represents the structural property of a system. It can be interpreted as the total number of terms in $i$ th grouping, $I_{i j}$ represents the total number of terms in the $j$ th sub-group of $i$ th grouping. In case there is no sub-grouping, then $I_{i j}$ is the same as $I_{i}$; the sub-groupings are arranged in decreasing order of size (i.e., number of elements in a loop). In general, two OOS products may not be isomorphic from the viewpoint of the architecture of sub-systems and interactions among sub-systems. A comparison is also carried out on the basis of the coefficient of similarity. The coefficient is derived from the structure, i.e., VPF - OOS and it compares two OOS products or a set of OOS products on the basis of similarity or dissimilarity. If the value of distinct terms in the $j$ th sub-grouping of the $i$ th grouping of VPF - OOS of two OOS products under consideration are denoted by $I_{i j}$ and $I_{i j}^{\prime}$, then two criteria are proposed as follows (Liu et al., 2004): The coefficient of similarity and dissimilarity are calculated using the number of terms only.

Criterion 1: The coefficient of dissimilarity $C_{d-1}$ based on criterion 1 is proposed as:

$$
C_{d-1}=\left.\left.\frac{1}{X_{1}}\right|_{i}\right|_{j} \eta_{i j}^{\prime}
$$

where

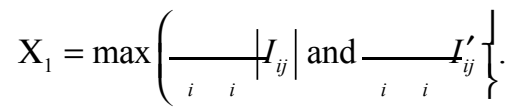

When sub-groupings are absent then

$$
I_{i j}=I_{i}, I_{i j}^{\prime}=I_{i}^{\prime} \text { and } \eta_{i j}=\left|I_{i j}^{2}-I_{i}^{\prime 2}\right| .
$$

When the sub-groupings exists then:

$$
\eta_{i j}=\left|I_{i j}^{2}-I_{i}^{\prime 2}\right| .
$$


Criterion 1 is based on the sum of the difference in the number of terms in different sub-groups and groups of VPF - OOS of two structurally distinct OOS architectures. There may be a case when some $\quad \eta_{i j}$ is zero though two systems are structurally different. This situation may arise when some of the differences are positive while some other differences are negative, such that I $\eta_{i j}$ become zero. To improve the differentiating power, another criterion is proposed $j$

Criterion 2: The coefficient of dissimilarity $C_{d-2}$ is proposed as:

$$
C_{d-2}=\frac{1}{X_{2}} \mathbf{I}_{i} \mathbf{I}_{j} \eta_{i j}^{\prime}
$$

where

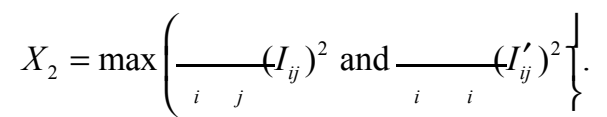

When sub-groupings are absent then:

$$
I_{i j}=I_{i}, I_{i j}^{\prime}=I_{i}^{\prime} \text { and } \eta_{i j}^{\prime}=\left|I_{i}^{2}-I_{i}^{\prime 2}\right|
$$

when the sub-groupings exists then:

$$
\eta_{i j}^{\prime}=\left|I_{i j}^{2}-I_{i j}^{\prime 2}\right| \text {. }
$$

Criterion 2 is based on the sum of the squares of the difference in the number of terms in different sub-groups and groups of VPF - OOS of two structurally distinct architectures of OOSs. It shows that $\eta_{i j}^{\prime}$ (criterion 2) is much larger than $\eta_{i j}$ (criterion 1). To increase the differentiating power further, another criterion 3 is proposed.

Criterion 3: The coefficient of dissimilarity $C_{d-3}$ based on criterion one is proposed

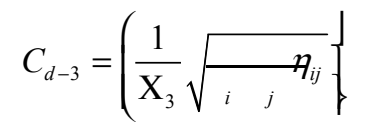

where $\eta_{i j}$ the same as is described in criterion 1 and

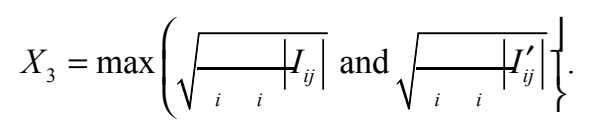

When sub-groupings are absent $I_{i j}=I_{i}$ and $I_{i j}^{\prime}=I_{i}^{\prime}$. Criterion 3 is derived from criterion 1 .

Criterion 4: The coefficient of dissimilarity $C_{d-4}$ based on criterion two is proposed

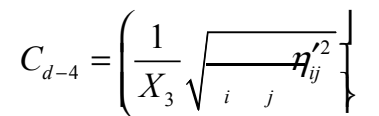

where $\eta_{i j}^{\prime}$ the same as is described in criterion 2 and 


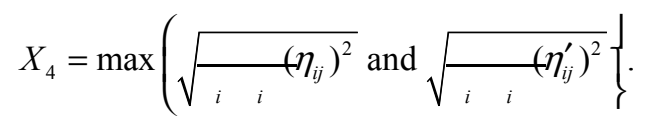

When sub-groupings are absent $I_{i j}=I_{i}$, and $I_{i j}^{\prime}=I_{i}^{\prime}$. Criterion 4 is derived from criterion 2 . This can further increase the differentiating power. Using above equations the coefficient of similarity is given as

$$
C_{m-1}=1-C_{d-1} ; \quad C_{m-2}=1-C_{d-2} ; \quad C_{m-3}=1-C_{d-3} ; \quad C_{m-4}=1-C_{d-4}
$$

where $C_{m-1}, C_{m-2}, C_{m-3}$ and $C_{m-4}$ are the coefficients of similarity between two OOS architectures under consideration, based on criterions 1-4.

Using the above-mentioned criteria, a comparison of two or family of OOS architectures is carried out. Two OOS architectures are isomorphic or completely similar from a structural point of view, if the structural identification sets for the two systems are exactly the same. This means the number of terms/ items in each grouping/ sub-grouping are the exactly same. The structural identification set equation (8) for the system shown in Figures 2 and 3 is obtained by considering its structure graph and VPF - OOS as $/ 1 / 8 /(2 \times 5) /(2 \times 5+9) /(2 \times 3+2 \times 2) /$.

It may be noted that the coefficients of similarity and dissimilarity lie in the range between 0 and 1 . If two OOS architectures are isomorphic or completely similar, their coefficient of similarity is 1 and the coefficient of dissimilarity is 0 . Similarly, if two OOS architectures are completely dissimilar, their coefficient of similarity is 0 and the coefficient of dissimilarity is 1 .

\section{Step-by-step procedure}

A step-by-step methodology is proposed which can permit technology managers, strategic and marketing decision makers to improve the quality of their OOS products. This methodology will also help in identifying the various choices of available designs, depending upon interaction/interdependencies or information flow between systems and their sub-systems and so on. A generalised procedure for the complete design and analysis of OOS system architecture is summarised below:

Step 1: Consider the desired OOS according to business, strategic, functional and marketing goals. Study the complete system and its sub-systems, and also their interactions.

Step 2: Develop a decomposition model of the OOS Figure 1, considering its sub-systems and interactions, along with assumptions, if any.

Step 3: Develop a systems structure graph of the OOS in Figures 2 and 3 with sub-systems as nodes and edges for interconnection between the nodes.

Step 4: Develop the matrix representation (2) and multinomial representations equation (3) of the OOS.

Step 5: Evaluate functions/values of diagonal elements from the permanent functions of distinct sub-systems equation (4) of the composite and repeat Steps 2-4 for each sub-system. 
Step 6: Identify the functions/values of off-diagonal elements/interconnections at different levels of hierarchy of the OOS amongst systems, sub-systems, sub-sub-systems, etc.

Step 7: Calculate the OOS identification set. Carry out architectural similarity and dissimilarity with potential candidates to take appropriate decisions.

Following the above procedure, these sub-systems can be broken down into sub-sub-systems and different graphs, matrices, and permanent representations can be obtained. Depending upon the depth of analysis required, the process could be taken to the constituent level and further. In certain cases, it may be possible to evaluate $E_{i j} \mathrm{~s}$ experimentally or using available mathematical models. With the help of this data, a complete multinomial for the OOS can be evaluated. Using/available standard modules of OOS sub-systems (e.g., dyads and loops of different sub-systems) in the global market; designers can develop alternative designs of OOS products and carry out analysis and improvement of existing OOS products. Work is in progress to carry out performance analysis of any OOS architecture from different perspectives using the structural model presented in this article.

\section{Usefulness of the proposed methodology}

Different stakeholders are benefited by the proposed methodology as follows:

- The methodology is dynamic in nature as sub-systems/components and interactions, which appear as variables in different models, may be changed without any difficulty.

- Thus, the approach helps to express the OOS system in quantitative terms, which has more often been expressed in qualitative terms. The procedure also helps to compare different OOS systems in terms of its characteristics and rate them for particular applications.

- The proposed methodology is a powerful tool in the hands of the system analyst, designer, decision makers and developers.

- Using this and morphological chart/tree, the system analyst, decision makers and designer can generate alternative design solutions and select the optimum one.

- Similarly, this method can be exploited to improve quality and reduce cost and time-to-market in learning industry.

\section{Conclusions}

The following concluding remark highlights the contributions of the present study.

- The proposed OOS system architecture is developed using system methodology and the graph theoretic model. They represent its structural information, including its systems, their sub-systems and their interconnections.

- The OOS system's permanent function is a mathematical model characterising the structure of the OOS product and also helps one to determine the OOS system index. 
- The permanent function of the OOS system architecture at a particular level of hierarchy represents all possible combinations of its sub-systems. The terms of the permanent function not only represent different sub-sets of OOS system architecture but also guide the analysts, designer, developer, manager, decision maker and purchaser to generate large number of alternative design solution before selecting an optimum system.

- The proposed structural coefficients of similarity and dissimilarity and identification sets are useful models to select optimum sets of sub-systems up to the component level to finally achieve high quality OOS system architecture in less cost and time by comparing their structures. As proposed systems model gives complete information of the existing system, SWOT (Strength-Weakness-Opportunities-Threats) analysis and cause and effect analysis (Fishbone diagram/Ishikawa diagram) can be carried out effectively. This permits achieving cutting edge over its competitors.

- Research is in progress to correlate, quantitatively, the structure of the system with different performance parameters of OOS e.g., quality, reliability, etc.

- As it is an integrated systems approach, all the sub-systems up to the component level are modelled and evaluated to be used as inputs for diagonal elements at next higher level and so on. It can be inferred that, to get the structural performance level (i.e., permanent index) of the overall system, the structural performance level of each sub-system at the lower level needs to be calculated and substituted as diagonal elements of the variable permanent adjacency matrix at higher level.

- The proposed structural methodology is comprehensive enough to deal with different structural and performance issues of OOS system architecture at different levels of its life cycle.

- A generalised methodology is also proposed to model a system consisting of $N$ sub-systems and their interactions.

- Current ongoing research deals with the correlation of structural models with the desired performance parameters (quality, reliability, responsiveness, flexibility etc), design and development of new systems as an improvement of existing systems and critical analyses of failed systems. The outcome will be reported in future publications.

\section{References}

Börstler, J. (2002) Teaching and Learning OO, Extended Abstract, Department of Computing Science Umeå University, SE-901 87 Umeå, Sweden.

Bosch, J. (2000) Design and Use of Software Architectures, Addison-Wesley, Harlow, England.

Clements, P., Bachmann, F., Bass, L., Garland, D., Ivers, J., Little, R., Nord, R. and Stanford, J. (2002) Documenting Software Architectures: Views and Beyond, Addison-Wesley, Harlow, England.

Coad, P. and Yourdon, E. (1991) Object-Oriented Analysis, Prentice-Hall, New Jersey, USA.

Courtois, P.J. (1985) 'On time and space decomposition of complex structures', Com. of the ACM, Vol. 28, No. 6, June, pp.590-604.

Deo, N. (2004) Graph Theory with Applications to Engineering and Computer Science, Prentice-Hall of India Private Limited, New Delhi. 
Gandhi, O.P., Agrawal, V.P. and Shishodia, K.S. (1991) 'Reliability analysis and evaluation of systems', Reliability Engineering and System Safety, Vol. 32, pp. 283-305.

Jurkat, W.B. and Ryser, H.J. (1996) 'Matrix factorization and permanents', J. Algebra, Vol. 16, pp.1-26.

Kriokorian, H.F. (2003) Introduction to Object - Oriented Systems Engineering, Part 1, IT Professional, March-April, pp.38-42.

Kruchten, P. (1995) The 4+1 View Model of Architecture, IEEE Software, Vol.12, No. 6, pp.42-50.

Liu, H., Uhlherr, A. and Bannister, M.K. (2004) 'Quantitative structure -property relationships for composites: prediction of glass transition temperatures for epoxy resins', Polymer, Vol. 45, No. 6, pp.2051-2060.

Mohan, M., Gandhi, O.P. and Agrawal, V.P. (2003) 'Systems modeling of a coal-based steam power plant', Proceedings of Institution of Mechanical Engineers, UK,. Part A, Power and Energy, Vol. 217, pp.259-277.

Rumbaugh, J. (1994) 'Building boxes: composite objects', JOOP, Vol. 7, No. 7, November-December, pp.12-22.

Sacks, I.J., Ashmore, B.C. and Alesso, H.P. (1983) Systems Interaction Results from the Digraph Matrix Analysis of Watts bar Nuclear Power Plant High Pressure Safety Injection System, Lawrence Livermore National Laboratory, Livermore, USA., Report No. UCRI-53467 Vols. 1-2, pp.16-33.

Seghal, R., Gandhi, O.P. and Angra, S. (2000) 'Reliability evaluation and selection of rolling element bearings', Reliability Engineering and System Safety, Vol. 68, pp.39-52.

Shaw, M. and Garlan, D. (1996) Software Architecture-Perspectives on an Emergine Disipline, Prentice-Hall, New Jersey, USA.

Tagoug, N. (2002) 'Object-oriented system decomposition quality', Proceedings of the 7th IEEE International Symposium on High Assurance Systems Engineering (HASE'02), pp.230-235.

Upadhyay, N. (2004) Analysis and Design of Algorithms, S.K. Kataria and Sons, New Delhi, pp.230-245.

Wilkinson, M. and Byers, P. (1993) 'The engineering of complex systems', Computing and Control Engineering Journal, August, pp.187-189. 